\title{
Synopsis: Lyme Disease in Canada - A Federal Framework
}

\author{
Centre for Food-borne, Environmental and Zoonotic Infectious Diseases ${ }^{1 *}$
}

\begin{abstract}
The Federal Framework on Lyme Disease Act required the federal government to develop a Federal Framework on Lyme Disease. To do this, the Public Health Agency of Canada held a conference with a wide variety of stakeholders who shared their experiences with Lyme disease, and discussed current knowledge and research. A draft version of the Framework was publicly posted on Canada.ca for a 30-day public consultation period. The final report, Lyme Disease in Canada: A Federal Framework was released in May 2017. The Framework includes the three pillars of surveillance; education and awareness; and guidelines and best practices. Implementation will require the involvement and collaboration of all stakeholders and all levels of government, with the Government of Canada committed to collaborating with domestic and international partners to exchange best practices in the prevention, diagnosis and treatment of Lyme disease.
\end{abstract}

\author{
Affiliation \\ ${ }^{1}$ Public Health Agency of Canada, \\ Ottawa, ON \\ *Correspondence: maladie \\ lyme_disease@phac-aspc.gc.ca
}

Suggested citation: Centre for Food-borne, Environmental and Zoonotic Infectious Diseases, Public Health Agency of Canada. Synopsis: Lyme Disease in Canada - A Federal Framework. Can Commun Dis Rep. 2017;42(10):212-4. https://doi.org/10.14745/ccdr.v43i10a04

\section{Introduction}

The Federal Framework on Lyme Disease Act, which received Royal Assent on December 16, 2014, required the federal government to develop a Federal Framework on Lyme Disease that would bring a sense of cohesiveness to surveillance, education and awareness, and guidelines and best practices efforts. To inform the development of this Framework, the Public Health Agency of Canada, on behalf of the Minister of Health, held a conference with over 500 participants. Lyme disease patients, their families and others shared their experiences with Lyme disease, while Lyme disease experts provided information on current knowledge and research related to Lyme disease treatment, prevention, diagnosis and management. Additionally, to allow for further feedback and refinement, a draft version of the Framework was publicly posted on Canada.ca for 30 days. The full report, Lyme Disease in Canada - A Federal Framework, was released on May 30, 2017 and is available online (1). It is intended to be a first step in guiding concrete action in areas where the federal government has a role. This is a synopsis of the full report.

\section{Background}

Canada has approximately 40 species of ticks. Of these, only a few are capable of transmitting pathogens, including bacteria and viruses, which have the potential to cause human illness.

Lyme disease, the most common tick-borne illness in Canada, is caused by the bacterium Borrelia burgdorferi. This bacterium is transmitted to people through the bite of blacklegged ticks and western blacklegged ticks.
Lyme disease occurs mainly in or near areas where infected tick populations are established. Adult ticks are about the size of a sesame seed, and nymphal (immature) ticks are about the size of a poppy seed. This means that people may not even know that they have a tick attached to them. People engaging in occupational or leisure activities, such as camping and hiking near or in forested or semi-forested areas where infected ticks are found, are at a higher risk of bite transmission. Exposure to ticks can occur in other circumstances, such as gardening, golfing or dog walking, if these activities occur in locations where ticks are found. It should be noted that ticks carrying Lyme disease are active through much of the year; however, bites leading to human infection are much more common during the spring and summer months.

In 2016, 987 Canadians were newly confirmed to have Lyme disease (2). These cases have been steadily growing since Lyme disease became nationally notifiable in 2009 and, while advances in knowledge and diagnostics have occurred over time, there is likely some degree of underreporting. The federal government and provinces and territories continue to work together to identify where Lyme disease cases are occurring in Canada.

\section{Responsibility}

The Government of Canada plays a national leadership role in preventing and controlling the spread of disease by helping to reduce the risk to Canadians posed by infectious diseases. It fulfills this role by tracking and monitoring infectious disease threats, undertaking research, promoting healthy behaviours, 
brokering knowledge transfer, and facilitating research and innovation.

Several federal government departments and agencies are involved in addressing Lyme disease in Canada:

- Public Health Agency of Canada

- Canadian Institutes of Health Research

- Health Canada

- Department of National Defence / Canadian Armed Forces

- Parks Canada

In Canada, prevention and control of Lyme disease requires collaboration among all levels of government and non-governmental organizations. As guided by the provisions of the Canada Health Act, provinces and territories are primarily responsible for the delivery of both direct health care services and public health activities. Provincial and territorial public health authorities, and Indigenous public health authorities, undertake prevention and control activities specific to their own jurisdictions. This work is conducted, in some jurisdictions, in collaboration with universities and other professional and non-governmental organizations.

\section{Public health actions}

All stakeholders, including patients and their advocates, health care providers, and public health authorities, recognize the importance of evidence-based approaches to both public health and the practice of medicine. Similarly, many agree that additional research is needed to fill in evidence gaps that exist for Lyme disease prevention and control, diagnosis and treatment.

As a first step to addressing evidence gaps, the Government of Canada will allocate new funding to address research gaps for Lyme disease, and will continue to collaborate with domestic and international partners to exchange best practices in the prevention, diagnosis and treatment of Lyme disease.

Other new actions under the Framework align with the federal role as they relate to surveillance; education and awareness; and guidelines and best practices. Implementation will require the involvement and collaboration of Lyme disease patients, patient groups, health care providers, public health authorities, expert researchers, and federal, provincial and territorial governments.

\section{Surveillance}

Surveillance is essential to understanding the risk posed to Canadians and is the foundation of the public health approach. Surveillance for Lyme disease includes monitoring both the distribution and spread of ticks that carry the pathogen $B$. burgdorferi, and tracking human cases of the disease across the country.

Federal and provincial public health authorities will continue to build on surveillance activities through integration and dissemination of innovative methods and best practices for human surveillance.

In addition, collection of human surveillance data in Canada on people who do not meet the case definition for probable or confirmed Lyme disease, but who experience various symptoms consistent with Lyme disease or similar ailments, will be initiated.

The increase in the distribution and number of individuals affected by Lyme disease in Canada is having a financial impact on the health care system. An analysis of the costs associated with Lyme disease will be undertaken, including both direct and indirect costs, where possible. Additionally, a national tick-borne surveillance system will be developed that includes Lyme disease and other possible tick-borne infections.

\section{Education and awareness}

Efforts need to be strengthened to enhance Lyme disease educational efforts so that they are more effective and available to Canadians and front-line health professionals, in support of provincial and territorial governments and other efforts.

Strengthening stakeholder engagement and partnerships will be critical to successful education and awareness campaigns. The development of early detection/early diagnosis educational materials, with a focus on high risk groups, will be supported to assist front-line health professionals and public health authorities in the prevention and timely diagnosis of Lyme disease using an evidence-based and patient-centered approach. Given that prevention is key in the public health approach for infectious diseases, a national tick and Lyme disease education and awareness campaign will be developed, in collaboration with partners, to complement existing outreach efforts aimed at reducing the risk of contracting Lyme disease and inform early intervention, diagnosis and treatment.

\section{Guidelines and best practices}

Guidelines and best practices that are evidence-based and effectively targeted to reach specific groups will be critical to address Lyme disease. Elements of consideration include prevention, diagnostics, treatment and research.

Currently, the best way to protect against Lyme disease is to prevent tick bites, or if bitten, to minimize the likelihood of infection by removing the tick in a timely, effective manner (3). Prevention and awareness programs are implemented by local public health authorities and other health care/veterinary providers to raise awareness of the risks of Lyme disease and measures to protect against tick bites.

The diagnosis of early Lyme disease infection by a physician or nurse practitioner is primarily a clinical one, based on symptoms and supported by a history of possible tick exposure, including travel history. Diagnosis is limited by the fact that not all patients will present with symptoms in the early stages. Current laboratory tests, which look for antibodies, perform better for untreated, later-stage Lyme disease, after the patient has developed an immune response. Concerns about false negative tests results have led some patients to seek private testing. The development and availability of improved laboratory testing options may reduce the current practice where some patients seek laboratory testing in private, for-profit laboratories that may not be using standardized testing. It is recognized that specific and more sensitive tests for Lyme disease are needed. 
There remain evidence gaps which can be informed through further research on treatment options. For example, some people experience symptoms that continue more than six months following treatment, described by some physicians as post-treatment Lyme disease syndrome or post Lyme disease syndrome. Other patients experience various chronic symptoms consistent with Lyme disease or similar ailments, sometimes referred to as chronic Lyme disease, which is not recognized by the majority of the medical community in Canada. Adding to the confusion is that current treatment guidelines for Lyme disease, developed by medical and scientific professional organizations and based on the best available evidence known worldwide, are not uniform. In Canada, the Association of Medical Microbiology and Infectious Diseases Canada (AMMI Canada) has endorsed and promoted the use of the Lyme disease treatment guidelines developed by the Infectious Diseases Society of America (IDSA), which represents physicians, scientists and other health care professionals who specialize in infectious disease (4). As such, the IDSA guidelines are used by the broader medical community. However, there are a small number of front-line health professionals who follow guidelines developed by the International Lyme and Associated Disease Society (ILADS), a multidisciplinary medical society dedicated to the diagnosis and treatment of Lyme and its associated diseases. The treatment recommendations in the ILADS guideline are different from those in the IDSA, particularly with regards to antibiotic use. Further research on best treatment approaches is required.

Moving forward, the Government of Canada will work with international public health partners to share best practices, which in turn, will be shared with all stakeholders. Front-line health professionals and provincial laboratories will continue to be supported in the laboratory diagnosis of Lyme disease. Partners, including provincial and territorial health care regulatory authorities, will be consulted on innovative, evidence-based approaches to address the needs of patients and a Lyme disease research network will be established to build on existing research to fill in evidence gaps and engage with clinical experts, researchers, and patient groups.

\section{Conclusion}

To ensure ongoing efforts to address Lyme disease are evidence-based and that the Government of Canada continues making inroads at preventing and controlling the spread of Lyme disease, the Public Health Agency of Canada will review this Framework within five years of its publication.

\section{Acknowledgements}

The Public Health Agency of Canada would like to thank all those who contributed their time and expertise during the development of the Federal Framework on Lyme Disease.

\section{Funding}

The development of the Federal Framework on Lyme Disease was paid for by the Government of Canada.

\section{References}

1. Government of Canada. Lyme Disease in Canada: A Federal Framework. Ottawa: Government of Canada. May 2017. https://www.canada.ca/en/public-health/services/ publications/diseases-conditions/lyme-disease-canadafederal-framework.html

2. Surveillance of Lyme disease: reported number of Lyme disease cases [Internet]. Ottawa: Government of Canada; [modified 2017 Jul 4]. https://www.canada.ca/en/publichealth/services/diseases/lyme-disease/surveillance-lymedisease.html\#a2

3. Prevention of Lyme disease. Ottawa: Government of Canada. https://www.canada.ca/en/public-health/services/diseases/ lyme-disease/prevention-lyme-disease.html

4. Wormser GP, Dattwyler RJ, Shapiro ED, Halperin JJ, Steere AC, Klempner MS, Krause PJ, Bakken JS, Strle F, Stanek G, Bockenstedt L, Fish D, Dumler JS, Nadelman RB. The clinical assessment, treatment, and prevention of Lyme disease, human granulocytic anaplasmosis, and babesiosis: clinical practice guidelines by the Infectious Diseases Society of America. Clin Infect Dis. 2006 Nov 1;43(9):1089-134. Epub 2006 Oct 2. Erratum in: Clin Infect Dis. 2007 Oct 1;45(7):941. DOI (http://dx.doi.org/10.1086/508667). PubMed (https:// www.ncbi.nlm.nih.gov/entrez/query.fcgi?cmd=Retrieve\&db= PubMed\&list_uids=17029130\&dopt=Abstract). 